Ancient Religions in North Africa

According to Medieval Arab Sources

\title{
Les religions antiques en Afrique du Nord d'après les sources arabes médiévales
}

Mohamed Benabbès

\section{Université de Tunis}

moh.benabbes@gmail.com - https://orcid.org/0000-0001-6960-7984

\section{Fecha recepción 07/12/2020 | Fecha aceptación 09/06/2021}

\section{Abstract}

Although the information provided by mediaeval Arabic sources about the Pre-Islamic history of North Africa is often scant and confused, the description they give of the religious situation in this region in the 7 th century is not far removed from that given by the classical sources. The majority of the African-Roman population was Christianised, but the local tribal world was divided between those who adopted the Christian religion and those who retained their traditional polytheism. When they invaded North Africa, the Muslims did not seem to have given much importance to the religious beliefs of the conquered peoples, instead me-

\section{Résumé}

Bien que les informations fournies par les sources arabes médiévales concernant le passé pré-islamique de l'Afrique du Nord sont souvent fort maigres et pleines de confusions et d'amalgames, l'image qu'elles fournissent de la situation religieuse de l'Afrique du Nord au VII ${ }^{\mathrm{e}}$ siècle n'est pas assez éloignée de celle que nous donne la documentation classique gréco-latine. Si la majorité absolue de la population africo-romaine était christianisée, le monde tribal africain était, quant à lui, partagé entre ceux qui ont adopté la religion chrétienne et ceux qui ont conservé leur polythéisme traditionnel. Au moment de leur invasion au VII siècle, 
Monográfico | Les religions antiques en Afrique du Nord d'après les sources arabes médiévales

rely distinguishing between those who agreed to submit to the new masters and those who opposed them. Bound by treaties of alliance with Roman power, the Christianised tribes raised the banner of resistance, whereas the pagan tribes, already in open struggle against the regime in place, did not adopt a united front. Those who submitted, such as the Lawāta and many others, sat uneasily within the late legal framework devised by the 9th century fuqaha concerning the status of the dhimmi. It was solely to remedy this situation that the epithet maǧūs was applied to them, stripped of all its primitive connotations in connection with Persian Zoroastrianism.

\section{Keywords}

Antiquity, Christianity, Mağūs, Montheism, North Africa, Paganism, Religion les Musulmans ne semblent pas avoir accordé une grande importance aux attitudes religieuses des peuples conquis. La ligne de démarcation était entre ceux qui acceptent de se soumettre aux nouveaux maitres et ceux qui s'en opposent. Les tribus christianisées, liées par des traités d'alliances avec le pouvoir romain, ont brandi l'étendard de la résistance. Les tribus païennes, déjà en lutte ouverte contre le régime en place, n'avaient pas un seul mot d'ordre. Ceux qui se sont soumis, comme les Lawāta et bien d'autres, correspondent mal au cadrage juridique tardif conçu par les fuqaha du IX siècle pour le statut des dhimmi. C'est seulement pour les y ajuster, qu'on leur appliqua l'épithète mağūs, dénudé de toutes ces connotations primitives en rapport avec le zoroastrisme perse.

\section{Mots-clés}

Afrique du Nord, antiquité, christianisme, mağ $\bar{u} s$, monthéisme, paganisme, religion 
BIEN QUE LES INFORMATIONS FOURNIES par les sources arabes médiévales concernant le passé pré-islamique de l'Afrique du Nord sont souvent fort maigres et pleines de confusions et d'amalgames, il n'est pas anodin de les prospecter pour discerner la perception de leurs auteurs des différentes formes de religiosité chez les habitants de l'Afrique du Nord à la fin de l'Antiquité et au moment de la conquête musulmane de la région. Mais, il est indispensable de rappeler, tout d'abord, le caractère assez tardif de cette documentation ${ }^{1}$. Les textes les plus anciens qui nous sont parvenus ne remontent pas au-delà du IX $\mathrm{I}^{\mathrm{e}}$ siècle apr. J.-C. Souvent, le contexte de la conquête musulmane ne représente qu'un vague souvenir qui ne cesse de s'embrouiller à mesure qu'on s'éloigne des évènements. Outre ce caractère tardif, les sources arabes médiévales sur l'Islam précoce au Maghreb sont très variées et d'une historicité assez variable ${ }^{2}$. Dans quelle mesure, donc, l'image décrite par les sources arabes médiévales reflète-elle la situation religieuse du Maghreb à la fin de l'Antiquité ? Ces bribes d'informations peuvent-elles nous aider à restituer le puzzle religieux en Afrique au moment de la conquête?

1. Voir en dernier lieu sur le corpus des textes arabes médiévaux concernant la conquête musulmane du Maghreb A. Mkacher et M. Benabbès, La conquête arabe de l'Afrique romaine: Anthologie bilingue de textes arabes, Paris, 2020.

2. Les sources arabes sur le Maghreb pendant le Haut Moyen Age se répartissent essentiellement en trois catégories : d'abord les ouvrages historiques, chroniques de la conquête (Futūh) comme le Futūh al Buldān d'Al-Baladhuri, ou histoire proprement dite (Ta'rīh) comme le Ta'rīh Ifrīqiyya wa-l-Maghrib d'al-Raqīq, les plus anciens étant ceux de Halīfa Ibn Hayyāt et d' Ibn 'Abd Al-Hakam qui ont vécu au cours de la deuxième moitié du IX siècle ; ensuite, les ouvrages à caractère géographique qui offrent ou bien des descriptions d'ensemble des " routes et des provinces " (Kutub Al-masālik wa-l-mamālik) comme l'œuvre d'Al-Bakri, ou récits de voyage (Rihla) comme l'ouvrage d'Al-Tiğānī ; enfin, les traités de généalogie tribale (Kutub al$a n s \bar{a} b)$, comme le Kitāb ğamharat ansāb al-Arab d'Ibn Hazm (XI' siècle). 


\section{Le monothéisme chez les Berbères au VII ${ }^{\mathrm{e}}$ siècle : quelle réalité ?}

Rares sont les cas où les textes arabes médiévaux s'arrêtent, même sporadiquement, sur l'état religieux de l'Afrique du Nord au moment de la conquête musulmane. Cependant, dans leur vision de la population nord-africaine à l'époque, ils font une distinction assez nette entre deux entités différentes parmi ceux qui ont opposé une résistance quelconque aux avancées des Musulmans dans cette contrée. En effet, dans les récits de la conquête arabe du Maghreb, on trouve une dichotomie claire entre, les Chrétiens (Nașāāa), synonyme de Rum (Romains/ Byzantins) et les Berbères, qui désignent clairement les autochtones et particulièrement ceux qui appartenaient au monde tribal ${ }^{3}$. Mais, s'agit-il là d'une distinction établie sur une base ethnique plutôt que religieuse ? Et peut-on en tirer quelques conclusions sur la situation religieuse au Maghreb au moment de la conquête arabe?

Voyons d'abord quelques témoignages sur cette dichotomie. La mention simultanément des Chrétiens et des Berbères se faisaient souvent dans un contexte de coalition entre ces deux parties dans leur lutte commune contre les envahisseurs musulmans. Cette alliance entre le pouvoir byzantin en Afrique et les tribus berbères semble avoir été constante durant les différents épisodes de la conquête musulmane du Maghreb, en dehors de la Cyrénaïque et du désert tripolitain. D’ailleurs, de tels accords entre les tribus maures et le pouvoir romain en Afrique étaient connus depuis la très haute antiquité et ils ont persisté jusqu'à la fin de l'Afrique romaine ${ }^{4}$. Ces traités de foedus prévoyaient, entre autre, une assistance militaire mutuelle en cas de guerre.

C'est ce qu'il y eu semble-t-il à l'occasion du premier choc entre l'armée byzantine d'Afrique, assistée par les Berbères, et les troupes musulmanes en 647, près de Sufetula (Sbeïtla) ${ }^{5}$. De même, lorsque le conquérant arabe 'Uqba b. Nāfi' s'est lancé dans une expédition désastreuse vers la Numidie, il était affronté, avant d'être exterminé, par les Berbères assistés par les troupes byzantines stationnés en Numidie. C’est dans ce contexte là que les narrateurs des événements de la conquête font apparaitre cote à cote les Chrétiens et les Berbères.

L'auteur anonyme de Kitāb al-Istibșār rapporte que « lorsqu'il ('Uqba) était venu au Maghreb, il s'était préoccupé de Tahouda et de Bādis, qui étaient alors parmi les villes les plus importantes de la région, et y avait reconnu l'existence d'une forte garnison de Chrétiens

3. Sur la notion de "Berbère" chez les auteurs arabes médiévaux voir en dernier lieu : R. Rouighi, Inventing the Berbers. History and Ideology in the Maghrib, Philadelphia, 2019.

4. Sur les traités romano-berbères, R. Rebuffat, "L'investiture des chefs de tribus africaines », dans F. Vallet et M. Kazanski (éds.), La noblesse romaine et les chefs barbares du IIIe au VIIe siècle, Paris, 1993, 23-33 ; ou pour la période byzantine, G. Dagron, «Ceux d'en face. Les peuples étrangers dans les traités militaires byzantins ", Travaux et Mémoires du Centre de Recherches d'Histoire et de Civilisation de Byzance, 10, 1987, 207-232.

5. Y. Moderan, « Lee dossier des sources non musulmanes sur l'Exarque Grégoire et l'expédition arabe en Ifrîkiyya en 647-648 ", dans L.A. García Moreno et M.J. Viguera Molins (éds.), Del Nilo al Ebro, I. Estudios sobre las fuentes de la conquista islamica, Alcalá de Henares, 2010, 141-178. 
(Nașārā) et de Berbères $»^{6}$. De même, le récit de la mort tragique de 'Uqba b. Nāfi', en 683, au sud de l'Aurès, à la main des Berbères conduits par Kusayla, s'achève souvent par une citation attribuée au traditionniste Šahr b. Ḥawšab (m. vers 719) qui aurait dit : « je me suis renseigné auprès certains Successeurs à propos de ce groupe. Ils m’ont répondu qu'il s'agit de 'Uqba et ses compagnons, tués par les Berbères et les Chrétiens à Tahouda » ${ }^{7}$. Plus tard, quand Hassān b. Al-Nu'mān décida vers 696 de s'attaquer pour la première fois à Carthage, capitale des Romains en Afrique, il a dû affronter aussi bien l'armée byzantine que celle des Berbères. C’est du moins ce que rapporte l'historien Ibn 'Idārī qui raconte que le général arabe, «apprenant que les Chrétiens (Nașārā), soutenus par les Berbères, avaient réuni une armée considérable dans le district de Șaṭūra ", il alla les combattre ${ }^{8}$. On peut multiplier les exemples de cette corrélation distinctive entre les Chrétiens et les Berbères, dans les récits de la conquête ${ }^{9}$. Mais, en quoi elle nous éclaire sur les religions des populations africaines à cette époque ? En effet, si le nom exonyme "Berbères " est une désignation ethnique des autochtones d'Afrique du Nord, le terme Nașārā, quant à lui, portait une connotation religieuse évidente. En effet, le terme Nașāra $\bar{a}^{10}$, pluriel de Nașrānì désigne, dans le Coran, l'ensemble des Chrétiens, d'une façon générale. Etymologiquement, le mot semble être une transcription de l'ethnique Nazaréens, qui désigne, au premier sens, les adeptes de Jésus de Nazareth, même si le terme figure sur la liste des hérétiques stigmatisée par les Pères de l'Église au moins depuis le début du $\mathrm{V}^{\mathrm{e}}$ siècle ${ }^{11}$.

Les Chrétiens d'Afrique, au moment de la conquête, sont donc désignés par l'appellation coranique générique Nașārā. Mais, il faut souligner que cette perception simpliste du christianisme chez les Musulmans n'était pas propre à l'Afrique du Nord. C'était une connaissance basée quasi uniquement sur la tradition orale et n'avait pas accès aux documents écrits.

6. Anonyme, Kitāb al-istibṣār, vol. 1, 174-175. Voici ce que dit le texte arabe :

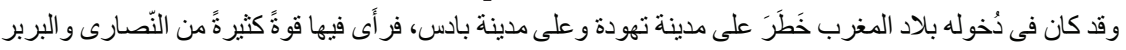

7. On trouve cette tradition, avec des variantes chez Abu-l-Arab, al-Māliki, al-Bekri, etc. Par exemple, on lit chez Abū al-Arab, Țabaqāt 'ulamā’ Ifrīqiyya wa-Tūnis, vol. 1 (édition et introduction par A. Chabbi et N. H.al-Baqi, $2^{\mathrm{e}}$ ed.), Tunis, 1985, 10 :

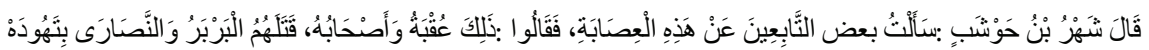

8. Ibn 'Id̄ārī, Kitāb al-Bayān al-Muğrib fī aḥbār al-Andalus wa-al-Mag̉rib, vol. 1 (édition critique par G.S. Colin et E. Levi-Provençal), Lieder, 1948, 42. Voici ce que dit le texte arabe :

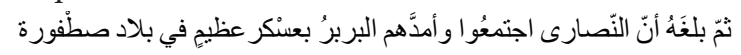

9. A. Mkacher et M. Benabbès, La conquête arabe..., op. cit., n. 1.

10. S. Griffith, "Al-Nașārā in the Qur'ān: a hermeneutical reflection », dans G. Said Reynolds (ed.), New Perspectives on the Qur'an. The Qur'an in Its Historical Context 2, London, 2012, 323-344; et aussi: F. De

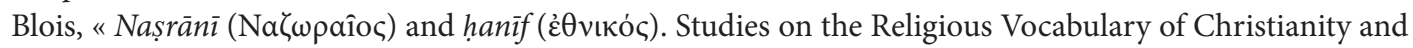
of Islam », Bulletin of the School of Oriental and African Studies, 65.1, 2002, 1-30.

11. Voir à titre d'exemple ce que Augustin, dans De haeresibus ad Quodvultdeum I 9 (composé en 428429), écrit à propos des Nazaréens : « Tout en reconnaissant que Jésus-Christ est le Fils de Dieu, les Nazaréens accomplissaient scrupuleusement les prescriptions de l'ancienne Loi, dont les chrétiens ont appris, à l'école des Apôtres, à comprendre le sens spirituel, et à délaisser l'observance charnelle ». 
Aucun renseignement n'est fourni, au passage, sur tout le débat christologique qui animait l'Eglise africaine dans sa lutte acharnée contre le monothélisme, au moment même où l'armée des Musulmans sonnait le glas de la présence byzantine en Afrique. Toutefois, la large diffusion du christianisme en Afrique au VII siècle est une réalité indéniable.

Elle touchait la majorité absolue des populations citadines et paysannes, de même qu'une bonne partie du monde tribal africain ${ }^{12}$. Par conséquent, la distinction entre les Chrétiens/Nașārā et les Berbères pourrait bien revêtir une différenciation ethnique sans aucune connotation religieuse, du moins pour les Berbères. Cependant, un autre détail pourrait semer le doute sur cette première constatation. En effet, les chroniqueurs de la conquête, qui restent muets quant à la religion des Berbères, font toujours exception, parmi ces derniers, pour les tribus ou les individus qui confessaient du christianisme et qui sont expressément désignés par l'appellation Nașārā. C’est le cas Berbères de Nafūsa, qui " avaient embrassé la religion chrétienne » et qui ont prêté assistance aux habitants des villes tripolitaines, notamment Sabratha, attaquée par les Musulmans, en $642^{13}$. C’est aussi le cas des « Berbères christianisés » (و على من أقام معهم على النّصر انية من الرّوم و البربر) qui furent assujettis par Hassān b. Al-Nu'mān moyennant leur payement d'un impôt foncier (Haräğg ${ }^{14}$. C'est enfin le cas des villes marocaines d'Aghmet ${ }^{15}$ et de Nafis ${ }^{16}$, qui abritaient des "Chrétiens Berbères » (نَصارى البرْبر), au moment de son siège par 'Uqba.

Ce sont pratiquement les seuls cas, dans les récits de la conquête du Maghreb, où la religion chrétienne des Berbères est mise en exergue. Examinons-les de prés. Pour les Nafūsa, tribus berbère qui habitait dans le Djebel qui porte encore le même nom en Libye actuelle, les traces matérielles de leur christianisation sont nombreuses et remontent au moins au $\mathrm{V}^{\mathrm{e}}$

12. Pour l'expansion du christianisme en Afrique avant de la conquête voir : Y. Modéran, "L'Église d'Afrique et la reconquista byzantine », dans J.-M. Mayeur, Ch. et L. Piétri, A. Vauchez et M. Venard (éds.), Histoire du christianisme, vol. 3, Paris, 1998, 699-717.

13. Al-Bakrî, Kitab al masalik wa-l-mamalik [Le livre des routes et des royaumes], vol. 2 (éd. critique en arabe par A. van Leeuwen et A. Ferré), Carthage, 1992, 656 : Le texte arabe porte :

$$
\text { البكري :افتنَح عمرو ابن العاص نفوسة وكانو انَصارى }
$$

14. Al-Raqiiq al-Qayrawān, Ta'rīh 'Ifrìqīya al-Mag̀rib (ed. critique par A.A. Zaydan et E.O. Musa), TunisTripoli, 1990, 15 : « Il instaura aussi un tribut du Haräğ sur ceux qui restèrent chrétiens parmi les Rūm et les Berbères ». Le texte arabe dit :

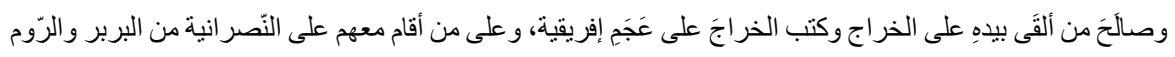

15. Ibn 'Abd al-Halīm, 'Ubayd Allāh b. Șāliḥ, Fath al-Arab li-l-Mag̉reb (éd. E. Lévi-Provençal), R.I.E.I.M., 2, 1954, 210 : «Il mit le siège devant Āgmāt et y trouva des Berbères chrétiens ». Le texte arabe porte :

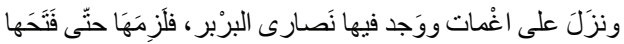

16. Anonyme, Kitāb al-istibșār... vol. 1, op. cit., n. 3, 208 : « Nāfĩs, ville qui remonte à une haute antiquité. 'Uqba b. Nāfi', Dieu l'ait en miséricorde, dirigea contre elle une expédition, y assiégea les Rūm et Berberes chrétiens et s'en rendit maître». Le texte arabe porte :

$$
\text { مدينةٌ قديمةُ أزليَّة غز اهَا عقبة بن نافع، رحمه الله، وحاصرَ ها وفيها الرَّوم والنّصارى البربر }
$$


siècle $^{17}$. Par contre, on est moins sûr pour les deux localités marocaines, Aghmet et Nafis, situées à plus de 350 kilomètres de Volubilis, où la pénétration du christianisme jusqu'à ces endroits assez éloignés est peu probable, pas moins d'ailleurs que la parvenue de 'Uqba dans ces régions ${ }^{18}$. Enfin, pour les Berbères christianisés et soumis au Harāğ par Hassan, il est difficile de déterminer leur pourcentage par rapport à la population totale. Il est fort probable que la majorité des tribus maures vivant à l'intérieur du Limes, à la lisière des villes et des campagnes africaines, étaient des chrétiens, longtemps déjà avant le VII ${ }^{\mathrm{e}}$ siècle.

Mais, les informations arides des sources arabes ne nous permettent pas d'entrevoir clairement cette approximation. On sent parfois, devant le silence complet de ces auteurs, qu'en dehors des cas mentionnés plus haut, les autres Berbères adhéraient à une autre forme de religiosité que le christianisme. Mais laquelle ? Peut-on penser à une autre forme de monothéisme comme le judaïsme?

Si la présence en Afrique de populations juives est attestée depuis une très haute Antiquité $^{19}$, nous ne disposons, au-delà du $\mathrm{V}^{\mathrm{e}}$ siècle, de presque aucun témoignage certain sur la présence du judaïsme en dehors peut-être de l'emblématique Doctrina Jacobi ${ }^{20}$ et d'un passage souvent sur-interprété de l'historien arabe Ibn Haldūn ${ }^{21}$ qui fait allusion à l'adoption, dans

17. Cf. J.B. Ward-Perkins et R.G. Goodchild, "The Christian Antiquities of Tripolitania », Archaeologia, 95, 1953, 35-37 ; A. Di Vita, « La diffusione del cristianesimo nell' interno della Tripolitania attraverso i monumenti e sue sopravivenze nella Tripolitania araba », Quaderni di Archeologia della Libia, 5, 1967, 121-142 ; D.J. Mattingly, Tripolitania, London, 1995, 211-212.

18. Beaucoup de doute règnent sur la réalité historique de cette poussée jusqu'à l'Atlantique. Voir A. Benabbès, «Les premiers raids arabes en Numidie Byzantine : questions toponymiques », dans $\mathrm{Cl}$. BriandPonsart (éd.), Identités et Cultures dans l'Algérie Antique, Rouen, 2005, 459-492.

19. Voir l'état de la question sur ce sujet dans C. Iancu et J.-M. Lassère (éds.), Juifs et Judaïsme en Afrique du Nord dans l'Antiquité et le Haut Moyen Age. Actes du Colloque International du Centre de Recherches et d'Études juives et hébraïques et du Groupe de Recherches sur l'Afrique Antique (Montpellier, 26-27 septembre 1983), Montpellier, 1985 ; et aussi, Y. Le Bohec, « Juifs et Judaïsants dans l'Afrique romaine. Remarques onomastiques ", Antiquités Africaines, 17, 1981, 209-229.

20. Doctrina Jacobi nuper baptizati dans G. Dagron et V. Déroche, « Juifs et chrétiens dans l'Orient du VII ${ }^{\mathrm{e}}$ siècle ", Travaux et Mémoires, 11, 1991, 17-248 (réédition dans Juifs et chrétiens en Orient byzantin, Paris, 2010).

21. Ibn Haldūn, Kitāb al-'ibar, vol. 6 (éd. par I. Chabbouh), Tunis, 2011, 140:

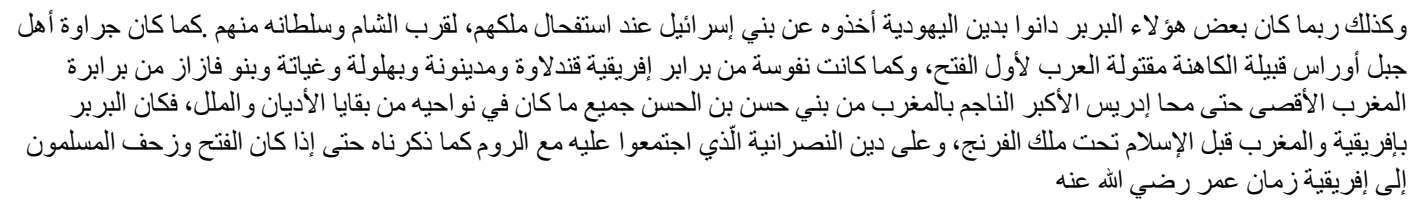

Traduction : « De même, peut-être certains groupements berbères avaient-ils même professé le judaïsme, empruntant cette religion aux Fils d'Israël au moment de l'apogée de l'extension de leur royaume, en raison de la proximité de la Syrie, alors toute puissante, de leur pays. Tel fut en particulier le cas des Djarâwa, qui habitaient les montagnes de l'Aurès, et qui formaient la tribu d'al-Kâhina tuée par les Arabes lors des pre-

Revista de historiografía 36, 2021, pp. 69-85 
une époque lointaine, de certaines tribus berbères du judaïsme. Si l'on peut admettre que certains groupements berbères aient professé le judaïsme pendant l'Antiquité et que certaines communautés juives aient survécu jusqu'au Moyen Age, il est irrecevable de généraliser la diffusion de cette religion en Afrique du Nord, en dehors d'une minorité communautaire éparpillée dans quelques villes et campagnes africaines ou chez certaines entités tribales ${ }^{22}$.

Donc d'après la littérature arabe médiévale, il semble bien qu'au moment de la conquête arabe de l'Afrique du Nord, l'empreinte chrétienne ou judaïque chez les populations berbères était relativement faible. Il semble donc que la majorité des berbères n'étaient pas des monothéistes, ni chrétiens ni juifs. Quelle étaient donc leurs croyances religieuses ? Comment étaient-ils qualifiés de point de vue de leur appartenance religieuse ? Etaient-ils considérés comme des païens ou des polythéistes ? ${ }^{23}$

\section{Le polythéisme chez les Berbères avant l'Islam et le problème de terminologie}

Le témoignage des auteurs arabes médiévaux sur la religion des Berbères pendant l'époque préislamique pose un sérieux problème de terminologie. Trois concepts différents sont utilisés pour décrire la situation religieuse des Berbères au moment de la conquête musulmane en Afrique du Nord. Il s'agit des termes Kuffār, mušrikūn et magūs. Chaque terme mérite une analyse particulière.

Le premier concept appliqué aux Berbères, même d'une manière assez limitée, est celui de Kuffār (ou Käfirūn), pluriel de Käfir, du verbe kafara qui veut dire " renier » au sens

mières invasions. Tel fut également le cas des Nafûsa, parmi les Berbères d'Ifrîqiya, et celui des Gandalâwa, des Madyûna, des Bahlûla, des Djiyâta et des Banû Fâzâz, parmi les tribus du Maghreb extrême. C'est Idris le Grand, descendant de Hasan b. al-Hasan, qui, se proclamant souverain au Maghreb, vint y effacer les dernières traces des différentes religions et confessions qui avaient subsisté [après l'islamisation]. En effet, comme nous l'avons déjà mentionné, les Berbères d'Ifrîqiya et du Maghreb étaient, avant l'Islam, sous la domination des Latins (Firandj), et professaient le christianisme, religion qu' ils partageaient avec Byzance (Rûm). Telle était la situation lorsque commencèrent les invasions musulmanes. Les Musulmans envahirent l'Ifrî̀iya sous le règne d'Omar (sic)-Puisse Dieu l'agréer! ”.

22. Sur la controverse à propos de la religion de la Kâhina et si elle était juive, chrétienne ou païenne, voir : Y. Modéran, Les Maures et l'Afrique romaine (IVe-VIIe siècles), Rome, 2003, 197-201 ; Y. Modéran, "Kahena ", Encyclopédie Berbère, 27, 2005, 4102-4111 ; A. Hannoun, Colonial Histories, Post-Colonial Memories. The Legend of the Kahina, Portsmouth, 2001.

23. Devrait-on utiliser ici le mot paganisme ou polythéisme ? On sait que le terme "païen » a été forgé par les pères de l'Eglise pour désigner la religion des Grecs et des Romains et la différencier de la leur et de celle des juifs, et que cette appellation a été synonyme de mauvaise croyance chez eux. Le terme polythéisme a été aussi forgé par le philosophe juif Philon d'Alexandrie pour désigner la religion des Grecs et des Romains, mais, il semble étymologiquement plus «neutre » que celui de paganisme. Sur cette question cf. F. Massa, " Nommer et classer les religions aux II-IV siècles : la taxonomie 'paganisme, judaïsme, christianisme" ", Revue d'Histoire des Religions, 234.4, 2017, 689-715. 
large, et plus particulièrement renier la divinité de Dieu ${ }^{24}$. Il est souvent traduit par «mécréants ». C'est là aussi un concept coranique appliqué d'abord aux polythéistes de la tribu Quraysh, avant d'être généralisé à tous les " infidèles " ou les " non croyants " par rapport à l' Islam ou à son Prophète ${ }^{25}$.

Le premier passage qualifiant les Berbères de mécréants (Kafara) est un texte attribué à l'historien kairouanais al-Raqīq qui rapporte que le calife 'Abd Al-Malik b. Marwān demanda à ses conseillers « qui serait la meilleure personne capable de venger la mort de 'Uqba sur les mušrikūn et les Berbères mécréants » (كفرة البربر)2 C'est ainsi qu’on lui indiqua Zuhayr b. Qays à qui il confia le gouvernement de l'Ifriqiya.

Le deuxième passage se trouve, avec quelques variantes, chez al-Raqīq, Ibn 'Iḍārī et alNuwayrī, dans un récit qui amplifie l'expédition douteuse de 'Uqba au Maghreb occidental en imaginant un discours qui aurait eu lieu entre le général arabe et un certain Julien ${ }^{27}$, présenté comme étant le gouverneur du district de Ceuta, au profit des Byzantins. Ce personnage aurait dit à 'Uqba, en le déconseillant de franchir le détroit de Gibraltar : «Il n'y a devant toi que les Berbères qui ressemblent à des brutes, car, ils n'ont jamais adopté le Christianisme ni toutes autres religions ; ils mangent des charognes, ils boivent le sang de leurs bestiaux vivants. Ce sont des gens qui ne croient pas en Dieu sublime (كفروا بالله العظيم), et ils ne le connaissent même pas. La majorité appartient à la tribu Mașmūda». ${ }^{28}$

En dépit de l'authenticité de ce dénigrement grossier des Berbères attribué au gouverneur chrétien de Ceuta, nous sommes là devant un des rares cas ou les auteurs arabes médiévaux fournissent quelques détails sur des pratiques et des coutumes alimentaires des Berbères en rapport avec leurs croyances religieuses. Mais, il semble que ce passage exprime davantage l'écho de quelques interdits alimentaires vétérotestamentaires, repris par l'Islam, et que les Berbères ne semblent pas respecter à cette époque. Il s'agit notamment de l'interdiction de boire le sang et de manger les animaux suffoqués et les charognes ${ }^{29}$. Nous savons que l'interdiction de la consommation des " animaux impurs », bien qu'elle ait été

24. S. Ghozi, Le concept de mécréance en terre d'islam : kufr, kafir, L'Harmattan, 2017.

25. W. Björkman, « Kāfir », Encyclopédie de l'Islam, 4, 1997², 407-409.

26. Al-Raqīq al-Qayrawān, Ta'rīh 'Ifrīqīya al-Maġrib..., op. cit., n. 14, 12 : فقال لهم :لا يصنُحُح للطّلب بدم عقبة من المشركين وكفرة البربر إلّاّ من هو مثنَلَهُ في دين الله .فاتَفق رأيُهم على زهير بن قيس البلوي

27. Sur ce personnage voir, Ch.-E. Dufourcq "Berbérie et Ibérie médiévales : un problème de rupture ", Revue Historique, 240.2, 1968, 293-324; et en dernier lieu : A. García-Sanjuán, La conquista islámica de la península ibérica y la tergiversación del pasado, Madrid, 2013.

28. Ibn 'Id̄ārī, Kitāb al-Bayān al-Mugigrib..., op. cit., n. 8, 32 :

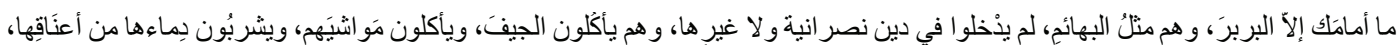

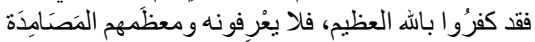

29. Nous lisons dans la Bible : "vous ne mangerez point la chair avec ce qui l'anime, son sang " Cf. $L a$ Bible. Torah, Nevihim, Ketouvim (trad. S. Cahen), Paris, 1994, 12. 
abolie par le Nouveau Testament ${ }^{30}$ est restée en vigueur au moins jusqu'au VII ${ }^{\mathrm{e}}$ siècle, par souci de lutte contre le paganisme.

C'était d'ailleurs l'objet d'un débat de grande actualité en Espagne visigothique au VII $\mathrm{VIII}^{e}$ siècles, entre juifs et chrétiens, comme le montrent les écrits d'Isidore de Séville ${ }^{31}$ et les Actes du VI $\mathrm{I}^{\mathrm{e}}$ concile de Tolède de $638^{32}$. Quel qu'en soit, ce passage perplexe reflète moins des croyances berbères au moment de la conquête, qu'un échantillon des griefs contre les Berbères, assez fréquents, dans les écrits arabes du Haut Moyen Age ${ }^{33}$.

Le deuxième terme avec lequel les auteurs arabes médiévaux qualifient les Berbères est celui de mušrikūn, pluriel de mušrik, celui qui pratique le širk. Etymologiquement, le participe actif pluriel mušrikūn provient du verbe ašraka, littéralement " associer », et qui désigne " ceux qui placent de faux dieux à côté du Dieu unique en leur rendant un culte qui n’est dû qu'à lui ». Le terme mušrikūn, est donc comme le terme Nașārā, un concept coranique qui sera rendu en français par « associateurs » ou « les associants », dans le sens de «polythéistes » ${ }^{34}$.

Le premier endroit où les Berbères furent qualifiés d'associateurs, se trouve chez l'égyptien al-Kindī, qui relate les expéditions de 'Uqba b. Nāfi' dans le désert libyen contre la confédération tribale des Lawāta. Défaits, ces derniers demandèrent alors la paix. C'est alors que 'Uqba aurait hésité à la leur accorder en répliquant que « dans nos traditions, les associateurs n’ont pas droit au pacte ». Il s'appuya ainsi sur un versé du Coran (IX 7) qui dit «Comment y aurait-il pour les associateurs un pacte? » et il ajoute : "C'est à moi de décider de votre sort, soit la paix, soit vous réduire en esclavage ${ }^{35}$. On reviendra plus loin au cas des Lawāta et leur traité.

Une autre mention des Berbères associateurs se trouve dans le récit de l'expédition qu'aurait fait 'Uqba b. Nāfi' en Numidie, chez l'historien al-Raqīq, qui, décrivant le sort des Byzantins et des Berbères suite à leur défaire devant l'armée de 'Uqba, écrivit : "lors de cette bataille, les plus vaillants guerriers et les nobles gens aussi bien des Rūm que des Berbères associateurs, furent anéantis $»^{36}$. Tous ces passages auraient pu nous indiquer un certain "polythéisme » des Berbères au moment de la conquête musulmane, si on ne trouve pas un usage du même adjectif mušrikūn appliqué d'une façon plus généralisée à tous les adversai-

30. Voir sur cette question : Nouveau Testament : Actes des Apôtres, 10,9-16 ; Epitre aux Corinthiens 10,2526 et Épitre aux Romains 14.

31. De fide catholica ex vetere et novo testamento contra Judaeos (Patrologia latina, vol. 83, col. 527).

32. Patrologia latina, vol. 84, col. 396.

33. Voir par exemple : M. Tilmatine, «L'image des berbères chez les auteurs arabes de l'époque médiévale ", Awal, 40-41, 2009-2010, 171 (Créer et transmettre chez les Berbères).

34. Le Coran (trad. et intro. D. Masson), Paris, 1967, cxi ; J. Berque, Le Coran. Essai de traduction de l'arabe, Paris, 1990.

35. A Kindī, Wulāt Miṣr, Bayrūt, 1959, 26. Le texte arabe dit:

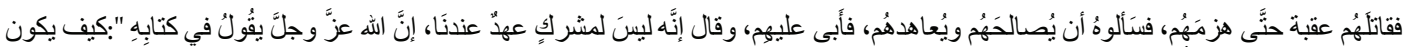

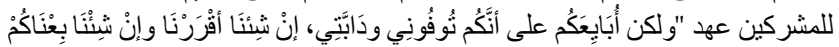

36. Al-Raqīq al-Qayrawān, Ta’rīh 'Ifrīqīya al-Maġrib..., op. cit., n. 14, 10 :

$$
\text { فقي تللك الوقُعة هلكَ رجال الرّوم و المشركين من البربر وفرسَانهم وأشر افِهم }
$$


res des Musulmans sans distinctions entre Chrétiens et Berbères. C'est le cas chez Ibn 'Idārī qui rapporte à propos de la bataille de Sbeïtla de 647 que « les Musulmans n'était qu'un petit nombre, tandis que les associateurs (mušrikūn) étaient au nombre de $120000 »^{37}$. D'ailleurs, dans le passage d'al-Raqīq indiqué plus haut, à propos du successeur de 'Uqba, et qui évoque "les mušrikūn et les kafara des Berbères » (المشركين وكفرة البربر ), nous ne sommes pas certains si l'épithète mušrikūn se rapportent forcément au mot «Berbères » ou à un autre nom sous-entendu, comme par exemple les Rūms ou les Chrétiens, car ces derniers aussi auraient pu être désignés par le terme mušrikūn.

Mais, cette confusion n'est pas propre à ces chroniqueurs de la conquête. Elle est inhérente à la perception musulmane du christianisme. En effet, les musulmans accusent les chrétiens d'adorer la croix et donc d'être, par ce fait, des associateurs si ne sont pas des idolâtres. Un passage d'al-Wāqidī dont le fameux ouvrage sur la conquête de l'Ifriqiya est malheureusement perdu, fut transmis de deux façons différentes mais révélatrices par deux auteurs tardifs. Le passage relate l'expédition de Hassān b. Al-Nu'mān contre Carthage en 697.

Al-Dahabi (m. vers 1352), dans son histoire de l'Islam rapporte d'après al-Wāqidī que Hassan il parvint à conquérir la ville de Carthage, dont les habitants étaient, à l'époque, «des $R \bar{u} m$ chrétiens, adorateurs de la croix $»^{38}$. Le même détail, rapporté par son contemporain Ibn Katir (m. 1373), toujours d'après al-Wāqidī, devint : " il parvint à conquérir la ville de Carthage, dont les habitants, à l'époque, étaient des Rūm, adorateurs des idoles ${ }^{39}$.

On voit ainsi donc que le terme « associateurs» ou associant n'est pas aussi clair dans la détermination de la situation religieuse des Berbères à la veille de la conquête musulmane.

Mais, si l'adjectif musrikun, comme on vient de le voir, est parfois commun aux Chrétiens et aux Berbères, il y a un autre qualificatif religieux qui fut appliqué, en Afrique du Nord, exclusivement aux Berbères. Il s'agit du terme mağ $\bar{u} s$, qui pose, quant à lui d'énormes problèmes méritant d'être analysés séparément.

\section{La question du « magisme " berbère}

Le terme de mağūs (mages) revient souvent chez les auteurs arabes médiévaux dans description des Berbères au temps de la conquête.

37. Ibn 'Id̄ārī, Kitāb al-Bayān al-Muǵrib...,op. cit., n. 8, 17 :

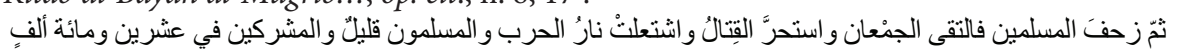

38. Al-Dahabī, Ta’rīh al-Islām wa-wafayāt al-mašāhīr wa-al-a lām, vol. 5 (éd. Baššār 'Awwād Ma'rūf, AlTab'a 1), Beyrouth, 2003, 67 :

$$
\text { فافتتح قرطاجنة، وأهلُها إذ ذالكَّ رومُ عُبَّادُ صليبٍ (الذهبي) }
$$

39. Ibn Katīr, Al-Bidāya wa-l-Nihāya, vol. 12, Le Caire, 2001, 142 :

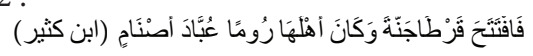


Dans les récits de la conquête, le terme mağūs est appliqué primordialement aux Berbères du Maghreb occidental, autrement-dit l'antique Maurétanie Tingitane et ses confins sahariennes. C'est le cas d' Ibn 'Id̄ārī, qui rapporte que «'Uqba est sorti pour envahir les Rūm et les Berbères, qui étaient, à l'époque, des adeptes du magisme et du christianisme ${ }^{40}$.

Encore plus précis, Ibn Hualdūn, évoque nommément les Șanhāğa, porteurs de voile, d'être au moment de la conquête "des mağ $\bar{u} s$, et n'avaient jamais adopté la religion chrétienne $»^{41}$. Ce qualificatif est appliqué par le même auteur à d'autres tribus berbères du Maroc comme les Walìlī (sur le Zarhun) et les Masmūda (dans le pays du Sūs) qui professaient tous "la religion des mağuss, et ne sétaient pas convertis à la religion des chrétiens " ${ }^{42}$

Cependant, d'autres passages d'Ibn Haldūn laissent entendre que l'adoption $d u$ « magisme » n'était pas propre aux Berbères de la partie occidentale de l'Afrique du Nord. L'auteur de Kitāb al-'ibar ( "Livre des Exemples»), affirme que la religion des Berbères était, sauf exception, celle des mağùs. Il écrivit dans ce sens : " Depuis le Maghreb jusqu’à Tripoli, ou, pour mieux dire, jusqu'à Alexandrie, et depuis la Mer Romaine jusqu'au Bilād al-Sūdān, toute cette région a été habitée par la race berbère, et cela depuis une époque dont on ne connaît ni les événements antérieurs ni même le commencement. La religion de ce peuple, comme celle de toutes les nations étrangères vivant en Orient comme en Occident, était la religion des mağūs. Il arrivait, cependant, de temps à autre que les Berbères se convertissent à la religion de leurs vainqueurs $»^{43}$. A en croire ce texte, on doit admettre que les Berbères, dans l'ensemble du Maghreb pré-islamique, étaient majoritairement mağ $\bar{u} s$.

Dans un autre passage plus explicite, Ibn Haldun résume la situation religieuse des Berbères avant l' Islam en affirmant qu'il y en a parmi eux ceux qui ont adopté le judaïsme ou le Christianisme, tandis que d'autres sont des mağ $\bar{s} s$, adorateur du soleil, de la lune et des idoles ${ }^{44}$

Examinons maintenant la portée du terme mağ̄ùs dans ces écrits arabes médiévaux sur le Maghreb. En effet, le terme «mağūs est employé originellement pour désigner précisément les zoroastriens perses, communément appelés les 'adorateurs du feu' $»^{45}$. Mais le terme aussi s'appliquait très tôt aux mazdéens, adeptes du mazdéisme, religion traditionnelle de l'Iran

40. Ibn 'Id̄ārī, Kitāb al-Bayān al-Muġrib..., op. cit., n. 8, 29:

$$
\text { فخرج، رحمة الله عليه، غازيًا للرّوم و البربر، و هم إذ ذاك مجوسُّ ونَصسارى }
$$

41. Ibn Haldūn, Kitāb al-'ibar..., op. cit., n. 21, 142

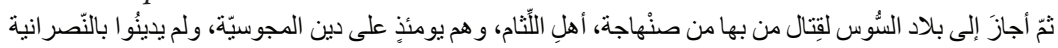

42. Ibn Haldūn, Kitāb al-'ibar..., op. cit., n. 21, 237 :

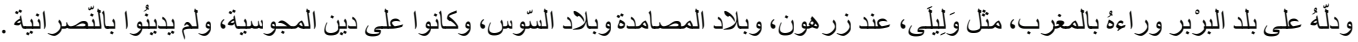

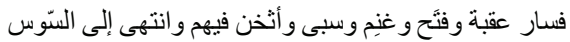

43. Ibn Haldūn, Kitāb al-'ibar..., op. cit., n. 21, 139 :

$$
\text { وكان دينُهم دينُ المجوسيّة، شأن الأعاجم كلّهم بالمشرق و المغرب إلّا في بعض الأحايين يَدينون بدينِ من غَلَب عليهم من الأمم }
$$

44. Ibn Haldūn, Kitāb al-'ibar..., op. cit., n. 21, 142 :

$$
\text { تاريخ ابن خلدون } 62142 \text { وكان منهم من تهوّد ومن تتصر وآخرون مجوسا يعبدون الثمس والقمر والأصنام }
$$

45. Cf. A. Melvinger, « al-Madjūs », Encyclopédie de l'Islam, 5, 1972, 115. 
ancien, qui, à la fin de l'empire perse, a connu l'introduction du culte des astres, sous l'influence des croyances chaldéo-babyloniens ${ }^{46}$.

Le Coran mentionne les mağūs sans préciser lequel de ces deux courants est désigné. Il les met, dans une même liste avec les juifs, les chrétiens, les sabéens $\left(s \bar{a}_{b} i^{3} a\right)^{47}$ et les associateurs $^{48}$, ce qui va diviser plus tard, les jurisconsultes musulmans quant au statut des mağ $\bar{u} s^{49}$ et s'ils relevaient ou non des "Gens du Livre ", c'est-à-dire les Juifs et les Chrétiens, théoriquement les seuls autorisés à conserver leurs propres confessions sous la domination de l'Islam, moyennant le paiement d'un impôt (la ğizya). Voyons maintenant si l'application du terme mağūs aux populations berbères avait-elle quelque crédibilité historique, ou si le mot a-t-il pris, avec le temps, d'autres acceptions.

Nous commençons d'abord par chercher d'éventuels liens ou traits communs entre le paganisme classique connu en Afrique du Nord antique et ce qu'on connait sur le "magisme " oriental. Plusieurs indices confirment l'existence bel et bien d'un culte astral parmi les croyances des Berbères à l'époque pré-islamique. Hérodote rapporte que tous les Libyens, ou presque, sacrifiaient au Soleil et à la Lune. ${ }^{50}$ Diodore de Sicile fait aussi une allusion au culte d'Hélios et de Séléné chez les Atlantes, c'est-à-dire le peuple qui réside aux environs du mont Atlas ${ }^{51}$.

Les sources archéologiques et épigraphiques viennent appuyer, à leurs tours, ces informations. De nombreuses représentations de Sol et de Luna décorent non seulement les stèles libyques, mais aussi les parois des grottes, des hypogées, et des dolmens protohistoriques au Maghreb ${ }^{52}$. La forte présence des disques solaires et des symboles lunaires sur les stèles de Baal Hammon/Saturne africain prouve que ce dieu aurait récupéré à son profit quelques aspects d'un ancien culte astral berbère ${ }^{53}$. Toutefois, il ne faut pas trop exagérer l'importance de ce dans le paysage religieux de l'Afrique antique ${ }^{54}$. Nous savons, d'autre part, que l'Afrique a connu aussi le culte mithriaque, profondément entachés par des influences iraniennes et chaldéennes, surtout en ce qui concerne

46. F. Lenormant, La magie chez les Chaldéens et les origines accadiennes, vol. 1, Maisonneuve, 1874.

47. Sur la nature des sabéens, plusieurs hypothèses ont été formulées : voir M. Tardieu, « Sabiens coraniques et 'Sabiens' de Harran », Journal asiatique, 274, 1986, 1-44.

48. Coran XXII 17 : «Le Jour de la Résurrection, Dieu distinguera les uns des autres : les croyants, les juifs, les sabéens, les chrétiens, les mages et les polythéistes - Dieu est témoin de toutes choses » :

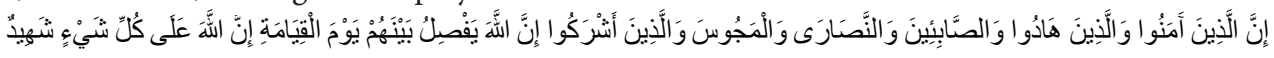

49. Pour Ibn Kat̄īr, ils font effectivement partie des Gens du Livre : Ibn Kat̄īr, Tafsīr, commentaire Cor. II 62. Pour Abū Yūsuf (m. 182/798) et al-Šaybānī (m. 189/805), ils étaient astrolâtres et ne relèvent pas de cette catégorie.

50. Hdt., IV 188.

51. Diod., III 57.

52. A. Cadotte, La romanisation des dieux : l'interpretatio romana en Afrique du Nord sous le Haut-Empire, Boston et Leiden, 2007, 366.

53. A. Cadotte, La romanisation des dieux..., op. cit., n. 52, 374.

54. Voir sur ce point le dossier archéologique rassemblé par L. Ben Abid, « Le culte du Soleil dans les provinces romaines d'Afrique ", L'Africa Romana, 19, 2012, 2333-2364. 
les dimensions sidérales ${ }^{55}$, même si ce culte était diffusé essentiellement chez les militaires ${ }^{56}$. N'estce pas à ce culte astral, mêlé au paganisme traditionnel, que fait allusion Ibn Haldūn en parlant du mağūs adorant le Soleil, la lune et les idoles ? Par sa connaissance encyclopédique, il est fort probable qu' Ibn Haldūn ait une idée assez bonne des croyances astrales des anciens Zoroastriens ou leurs descendants les Sabéens. Mais, il n'est pas impossible non plus que son usage du terme mağūs ici est dans un sens plutôt générique pour désigner les « païens » indépendamment de leurs croyances et des dieux qu' ils adorent.

Que ces croyances "païennes ", largement diffusées chez les Berbères pendant toute l'Antiquité, aient survécu jusqu'au VII ${ }^{e}$ siècle et au-delà, rien n'est étonnant même si on en manque de témoignages évidents $s^{57}$. On a parfois supposé que le polythéisme se soit prolongé, en Afrique, jusqu’à une époque tardive, en se fondant sur le maintien dans les épitaphes tardo-antiques de formules païennes $(D[i i s] M[\text { anibus }] S[\text { acrum }])^{58}$. Mais, la persistance du paganisme semble être encore plus manifeste chez les tribus berbères, en particulier en Tripolitaine et en Cyrénaïque, comme le montre, au début du V siècle, Synésios de Cyrène. Cet évêque de Ptolémaïs rapporte que, dans la plaine de Barké (Barqa), « les barbares ont brûlé des églises et ils ont profané une table d'autel en en faisant un étal de boucherie et en y découpant des viandes". Il ajoute que ces même Barbares " emportent des églises les objets mystiques utilisés pour la liturgie et le service du Seigneur afin de les offrir à leurs divinités ${ }^{59}$. Au $\mathrm{VI}^{\mathrm{e}}$ siècle encore, Corippe (V 12-26) constate aussi que les Laguatan (les Lawāta) adoraient toujours un dieu barbare, Gurzil, fils d'Ammon Corniger, représenté sous forme d'idoles en bois et en métal. Ces mêmes populations célèbrent également d'autres cultes païens dans des temples, en particulier à Augila, avant que la ville ne fusse occupée par les Byzantins, qui au dire de Procope, détruisirent le sanctuaire d'Ammon, et construisirent à sa place une église dédiée à la Théotokos ${ }^{60}$. Nous sommes là au milieu du VI siècle, et la puissante confédération tribale berbère, les Levathae (Lawāta), était toujours païenne. Est-ce que les Byzantins ont-ils réussi à évangéliser toutes les populations autochtones de la Cyrénaïque et de la Tripolitaine ? $\mathrm{Ou}$ au contraire ces tribus ont conservé leurs croyances polythéistes jusqu'au VII siècle?

Il est difficile de répondre à ces questions d'autant plus que les textes arabes médiévaux, exception faite pour les Nafoussa, ne livrent aucun détail qui permettrait de connaitre la religion de ces tribus berbères. Mais le silence absolu de ces textes sur une éventuelle christianisation des Lawāta en 642 autorise à conclure qu'ils étaient perçus comme des païens,

55. CIL VIII 2675 (Lambèse, III' s. ap. J.-C.) : Deo Sol(i) In/victo Mi/thrae. / M(arcus) Aurel(ius) / M(arci) f(ilius) Sergi/a Carnu/nto Sabi/nus praelfect(us) leg(ionis) / III Aug(ustae) P(iae) V(indicis) / [[Maximi]]/ [[nianae]] $v$ (otum) $s$ (olvit) l(ibens) a(nimo). De même CIL VIII $18025 ; 21523$, etc.

56. L. Bricault, «Les dieux de l'Orient en Afrique romaine », Pallas, 68, 2005, 289-309.

57. Voir sur la persistance du paganisme en Afrique les remarques de M. Le Glay, Saturne Africain. Histoire, Paris, 1966, 424.

58. N. Villaverde Vega, Tingitana en la Antigüedad Tardía. Siglos III-VII d.C., Madrid, 2001, 321-322.

59. Synes., Catastase II (éd. N. Terzaghi, Rome, 1944, 290).

60. Procop., De Aedif. VI 2, 19. 
même si le statut fiscal qui leur fut imposé, devrait théoriquement prouver un raisonnement différent ${ }^{61}$. En effet, les chroniques de la conquête rapportent que le conquérant 'Amr b. al'Āṣ a conclu un traité de reddition avec les Berbères Lawāta, habitants de la Pentapole, qui s'engagèrent à payer une ğizya de treize mille dinars «ils vendraient au besoin, pour s'en acquitter, leurs propres enfants $\gg .{ }^{62}$

Or, nous savons que pour certains exégètes du Coran du moins, ce type de pactes n'est réservé qu'aux "Gens de Livres » et pas aux polythéistes ${ }^{63}$. En effet, Ahl al-kitāb, c'est-à-dire les adeptes des autres religions monothéistes, essentiellement les juifs et les chrétiens, doivent reconnaître leur situation d'infériorité, en acceptant de verser la taxe de capitation. A cette condition, ils peuvent conserver leurs religions en obtenant le droit de vivre en paix en tant que sujets bénéficiant de la protection de l'État musulman. Quant aux polythéistes, ils sont placés devant l'alternative de la conversion ou la mort.

Mais, dans la pratique, les premiers musulmans nont pas respecté scrupuleusement cette interprétation. Plusieurs peuples ou communautés qui ne confessaient ni le Judaïsme ni le Christianisme furent pourtant assujettis moyennant leur acquittement de la ğizya $a^{64}$. Tel le cas, semble-til avec les Lawāta berbères. Pour justifier cette conduite, qui n'était pas d'ailleurs propre à l'Afrique du Nord, les jurisconsultes musulmans firent appel aux recueils de hadith pour chercher des analogies dans les dires ou les pratiques du Prophète de l' Islam ${ }^{65}$. C'est alors qu'on évoque une tradition transmise par Mālik b. Anas (m. en 179/790), par la voie d' Ibn Šihāb al-Zuhrī (m. en 124/742), qui enseigne que la ğizya fut perçue par Muhammad sur les mağūs du Bahreïn, par le calife 'Umar b. al-Hatțāb sur les mağūs Perses et par le calife 'Uțmān sur les Berbères ${ }^{66}$.

61. Cf. R. Brunschwig, «Ibn 'Abdalh'akam et la conquête de l'Afrique du Nord par les Arabes ", Annales de l'Institut d'Etudes Orientales, 6, 1942-1947, 111-113.

62. Voir par exemple, Al-Balāḍurī, Futūh Al-Buldān (éd.'Abd al-Amīr Sahnā), Bayrūt, 1992, 314; Ibn 'Abd Al-Hakam, Futūh Mișr wa-al-Mag̉rib (éd. 'Alī Muhammad 'Umar), Caire, 1995, 229.

63. Le texte coranique sur lequel s'est fondé le statut juridique des dhimmīs est le suivant (IX 29) : « Combattez ceux qui ne croient point en Allah ni au Dernier Jour (qui) ne déclarent pas illicite ce qu'Allah et son Apôtre ont déclaré illicite, (qui) ne pratiquent point la religion de Vérité, parmi ceux ayant reçu l'Écriture ! (Combattez-les) jusqu’à ce qu'ils paient la ğizya, directement (?) et alors qu' ils sont humiliés».

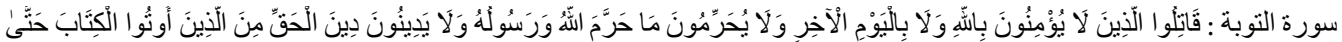

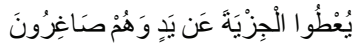

Comme il s'agit du seul passage dans lequel le mot ğizya est explicitement mentionné, son interprétation a posé beaucoup de problèmes aux exégètes et commentateurs du Coran.

64. Pour la question de la ğizya : A. Ziauddin, « The concept of jizya in early Islam », Islamic Studies, 14, 1975, 293-305.

65. Cf. R. Brunschwig, «Ibn 'Abdalh'akam...», op. cit., n. 61, 111-113.

66. Malek b. Anas, Al-Muwatta, Kitab al-Zakat, n 617 ; Al-Shafi' I, Al-Musnad, nº 1008.

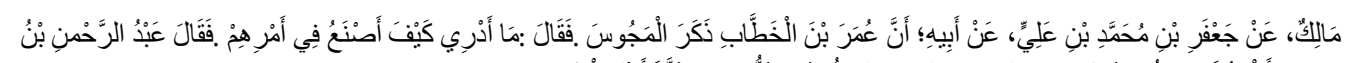

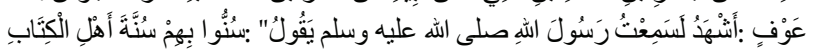

La même tradition est rapportée, par la même voie de Zuhri, par Saïd b. Ufayr. Voir Ibn Sallam, Le Kitâb al-Amwâl, p. 40 :

Revista de historiografía 36, 2021, pp. 69-85 
On s'appuyait, pour argumenter cette pratique, sur un hadith transmis aussi par Malik b. Anas, par la voie de 'Abd al-Raḥmān b. 'Awf, selon lequel le Prophète aurait déclaré au sujet des mağuss : "Traitez-les comme les Gens du Livre " ${ }^{67}$.

Lopinion de l'iman de Médine, à propos du statut des Berbères et leur assimilation aux mağūs, fut répandue par ses disciples égyptiens et surtout Kairouanais. Mais, au lieu d'homologuer le statut des Berbères avec celui des mağuss, comme le prescrive la tradition prophétique évoquée plus haut, on a fini par identifier les Berbères aux mağūs. Nous lisons dans la fameuse Mudawwanna de Sahnūn, (m. en 804), au livre de la guerre sainte (III 46) : «Mālik a dit au sujet des Berbères maǧūs (sic!) : 'Uțmān b. 'Affān a prélevé la ğizya sur eux ». Pourtant, Mālik n’a jamais parlé des Mağūs-Berbères. Il a parlé distinctement des Mağūs puis des Berbères. Interrogé au sujet des Fezzanais (al-Fazāzina) assujettis par 'Uqba b. Nāfi ${ }^{68}, M a \bar{l}$, ik répondit, toujours selon la version de Suhnun: « Je ne suis pas d'avis qu'on les combatte avant de les avoir invités à embrasser l' Islam. $S$ 'ils n'acceptent pas, on les invite à payer la ğizya tout en gardant leur religion ; s'ils répondent favorablement, on accepte cela d'eux $»^{69}$. Donc l'avis Mālik était clair : la prescription de la ğizya, en dehors des Gens de Livre, ne se limite pas aux mağūs, mais aux Berbères aussi, comme « elle s'applique également aux Slaves, aux Avars, aux Turcs et autres non-Arabes qui ne sont pas des gens du Livre ». Et Mālik de préciser : " tous ces peuples-là sont au même statut que les

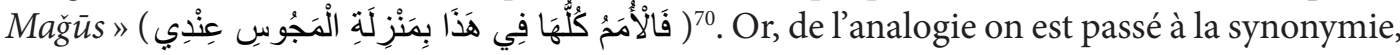
et par un glissement sémantique, on a fini par comprendre que tous les peuples, qui ne sont pas Juifs ou Chrétiens, sont des mağ $\bar{u} s$.

On comprend alors qu'au fur et à mesure de l'élargissement de l'Empire musulman, on déclarait mağùs tous les païens avec lesquels on désirait faire un accord, pour éviter toute discussion théorique ${ }^{71}$. Ça pourrait être-là l'explication la plus probable du qualificatif mağ $\bar{u} s$ appliqué, à l'ensemble ou à une partie des Berbères, au temps de la conquête musulmane.

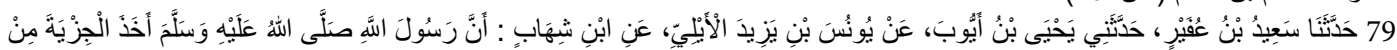

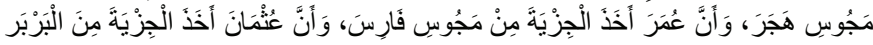

67. On lit dans le Muwatta' de Malik b. Anas le texte suivant :

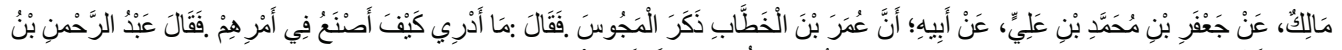

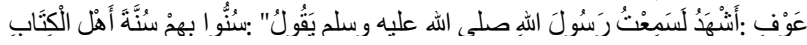

68. Ibn 'Abd Al-Hakam écrit à propos de l'expédition de 'Uqba b. Nâfi' vers 668 au Fazzān, dans son Futūh Mișr wa-al-Mağrib, p. 166 : "Quand il fut près de Jerma, capitale du Fezzan, il envoya des messagers demander aux habitants s'ils étaient disposés à se convertir à l'islam. Ils acceptèrent ».

69. Al-mudawwana al-kubrā lil-imām Mālik Ibn-Anas al-Așbahị, riwājat al-imām Sahnūn, vol. 1, 529 : "Ceci, conclut Suhnun, vous montre l'opinion de Mâlik au sujet de n'importe quel peuple ; car, ce qu' il a dit des Fezzanais s'applique également aux Slaves, aux Avars, aux Turcs et autres non-Arabes qui ne sont pas des gens du Livre».

70. Al-mudawwana al-kubrā lil-imām Mālik Ibn-Anas al-Așbahị, riwājat al-imām Sahnūn, vol. 1, 529.

71. On trouve la même opinion chez al-Auzầi (m. 157H/774), fondateur du rite qui a précédé le mâlikisme en Espagne. 


\section{Conclusion}

Pour conclure, on peut dire que l'image que fournissent les sources arabes médiévales, et particulièrement les récits de la conquête musulmane du Maghreb, sur la situation religieuse de l'Afrique du Nord au VII siècle n'est pas, au final, assez éloignée de celle que donne la documentation classique gréco-latine. Si la majorité absolue de la population africo-romaine était christianisée, le monde tribal africain était, quant à lui, partagé entre ceux qui ont adopté la religion chrétienne et ceux qui ont conservé leur polythéisme traditionnel. Au moment de leur invasion au VII ${ }^{\mathrm{e}}$ siècle, les Musulmans ne semblent pas avoir accordé une grande importance aux attitudes religieuses des peuples conquis ${ }^{72}$. La ligne de démarcation était entre ceux qui acceptent de se soumettre aux nouveaux maitres et ceux qui s'en opposent. Les tribus berbères christianisées, liées par des traités d'alliances avec le pouvoir romain, ont brandi létendard de la résistance. Les tribus païennes, déjà en lutte ouverte contre le régime en place, n'avaient pas un seul mot d'ordre. Ceux qui se sont soumis, comme les Lawāta et bien d'autres, correspondent mal au cadrage juridique tardif conçu par les fuqaha du IX siècle pour le statut des dhimmi. C'est seulement pour les y ajuster, qu'on leur appliqua l'épithète mağùs, dénudé de toutes ces connotations primitives en rapport avec le zoroastrisme perse.

72. Voir, dans ce même volume, Anis Mkacher, 87-106. 